\title{
Zinc sulfate treatment of vocal process granuloma
}

\author{
Taner Yılmaz
}

Received: 2 September 2012/ Accepted: 19 September 2012/Published online: 30 September 2012

(C) Springer-Verlag Berlin Heidelberg 2012

Dear editor,

I would like to make several comments on a manuscript by Sun et al. [1].

There is a mismatch between the abstract, text, and Table 1. According to the abstract, 11 patients had history of laryngeal trauma and 5 were idiopathic; 11 patients were operated before and recurred, 5 did not receive any treatment. According to the "Materials and methods", all 16 patients were traumatic; all were operated and recurred. Table 1 is the same as abstract. I accepted the text as correct.

The authors reported 16 patients with vocal process granuloma, which developed after intubation or external trauma to larynx. All patients were operated and all lesions recurred postoperatively. The authors prescribed $200 \mathrm{mg}$ zinc sulfate orally after meals, making a total daily dose of $600 \mathrm{mg}$, including $242 \mathrm{mg}$ zinc. All vocal process granulomas disappeared within 4-12 weeks after the treatment.

Zinc is an essential mineral that plays vital role in many biochemical reactions. Daily requirement for an adult is around $11 \mathrm{mg}$. Its deficiency leads to many diseases.

In head and neck surgery, we use zinc oxide cream to decrease skin inflammation around tracheotomy or tracheostoma and to decrease excess granulation tissue reaction in open wound care. Because of its anti-inflammatory effect in local use, it is pleasing to learn that it is helpful in the treatment of vocal process granuloma.

T. Yilmaz (ه)

Division of Laryngology and Phonosurgery,

Department of Otolaryngology-Head and Neck Surgery,

Faculty of Medicine, Hacettepe University,

06100 Ankara, Turkey

e-mail: tyilmaz@hacettepe.edu.tr
In medicine, there is a general rule saying that if there is no indication for a specific test or treatment, there is already a contraindication. If a mineral is to cure a disorder, it must be deficient in the body. For example, if anemia is to respond to iron treatment, iron must be really deficient in the body. Furthermore, iron supplementation does not cure other forms of anemia and is even contraindicated. If zinc is expected to cure vocal process granuloma, those patients must be deficient in zinc. If those patients have normal blood and tissue levels of zinc, zinc supplementation, even in excessive doses (the authors are giving 22 times the daily requirement), is not expected to cure the problem. Were their patients deficient in zinc? Did the authors measure blood or tissue levels of zinc before starting treatment with zinc sulfate? If no, do they think that their results are evidence-based?

I do not expect their patients to be deficient in zinc; because, they are in such a social level that they could reach medical services easily and were even operated. What do they think the mechanism of action of zinc is in curing vocal process granuloma, if it is not deficient in body? In vocal process granuloma there is no perfect cure using many conservative and invasive methods available. How can zinc sulfate give perfect cure of vocal process granuloma without any recurrence even 1 year after treatment?

\section{Reference}

1. Sun GB, Sun N, Tang HH, Zhu QB, Wen W, Zheng HL (2012) Zinc sulfate therapy of vocal process granuloma. Eur Arch Otorhinolaryngol 269(9):2087-2090 